\section{Camptocormie: Myopathie ohne Befund}

Bei der Camptocormie handelt es sich um eine schwer beeinträchtigende, durch muskuläre Schwäche bedingte Beugung des Rumpfes, die enorme Schmerzen verursacht und für die Patienten die oftmals zugrunde liegende Parkinson-Erkrankung (PD) nahezu in den Hintergrund treten lässt.

$D$ Arne Wrede, Homburg, untersuchte die Camptocormie (CC) bei Parkinson-Patienten mittels Muskelbiopsien. Dabei konnte er weder Ablagerungen von pathologischem $\alpha$-Synuklein innerhalb der Muskulatur als Auslöser des beschriebenen Schädigungsbildes identifizieren, noch fanden sich Hinweise für eine Myositis, eine Mitochondriopathie oder eine myofibrilläre Myopathie. Daher entwickelte Wrede die Hypothese, dass als Ursache einer CC bei PD eine propiozeptive Dysregulation vorliegt, zum Beispiel ein nerval gestörtes oder unzulängliches Feedback an das ZNS über den vorliegenden Muskeltonus. Diese fehlerhafte Information führt zu Fehlsteuerungen bei der erneuten zentralen Innervation der Muskulatur. Möglicherweise wird der Muskel nur noch selten oder nie in den Ruhetonus versetzt. Dies hat langfristig eine Myopathie zur Folge, die schließlich in Faserverlust oder -atrophie mündet. Muskelbiopsien zur weiteren Abklärung kön- nen unter Umständen sinnvoll sein, etwa um eine Myositis auszuschließen. Sie bedürfen aber einer besonderen professionellen Aufarbeitung. „Die klassische Fixierung des Biopsats in Formaldehyd ist in diesem Fall falsch", betonte Wrede.

\section{Rigor als Folge ausgeschalteter Hemmung}

Professor Günther Deuschl, Kiel, sieht als Hauptursache der CC eine Schwäche der paravertebralen Muskulatur. Ebenfalls hypothetisch führt ,der Rigor der paravertebralen Strecker zu einer Muskelzerstörung." Ursache des Rigors ist möglicherweise der Verlust der spinalen autogenen Hemmung von 1b-Fasern bei PD, die bei gesunden Menschen den Muskel vor mechanischer Überlastung schützt. Der hemmende 1b-Signalweg läuft über ein Interneuron, das auf das Motoneuron projiziert. Aus Versuchen weiß man, so Deuschl, dass bei gesunden Kontrollen im Falle einer Doppelreizung der zweite Input inhibiert wird, um die Muskulatur vor einer Überreizung zu schützen. Dieser Schutzmechanismus fehlt bei Parkinson-Patienten. Die Hemmung kann mittels tiefer Hirnstimulation (THS) wieder reaktiviert werden. Dies erklärt auch, warum die THS bei CC wirksam ist. „, $\mathrm{Al}$ lerdings nur solange die entsprechende Muskulatur noch nicht zerstört ist." schränkt Deuschl ein. Dies ist bei langzeitiger CC jedoch der Fall.

Ansonsten empfiehlt Deuschl aus seiner Erfahrung als mögliche Behandlungen einer CC in jedem Fall Physiotherapie. Ein neuartiges zweiteiliges Korsett konnte in einer kleinen Studie eine erhebliche Schmerzreduktion und Verbesserung der Lebensqualität bewirken. Ebenso hilft ein Hochrollator als Gehhilfe. Medikamentös scheinen lediglich Botulinumtoxin und Lidocain gewisse Effekte zu zeitigen. „Rucksäcke tragen haben wir bei fast jedem Patienten versucht, und haben eigentlich nie Erfolge gesehen." Nur sehr selten ist eine CC Dopa-sensitiv. „Bezüglich der DopaminAgonisten machen wir immer einen Auslassversuch, um zu sehen, ob die CC besser wird“, so Deuschl.

Reimund Freye

10. Deutscher Kongresses für Parkinson und andere Bewegungsstörungen, 4.-6.5.2017, Baden-Baden

\section{Zerebralparesen bei Kindern - Vertikalisierung durch Botulinumtoxin ermöglichen}

Wie früh sollte man Kinder mit Zerebralparesen behandeln? Sobald erkennbar ist, dass sie in ihrer motorischen Entwicklung retardiert sind? Neben funktionellen Verfahren gehört eine BTX-Gabe unbedingt in den Behandlungsplan.

$\mathrm{N}$ ach wie vor ist jedoch Botulinumtoxin erst ab dem zweiten Lebensjahr zugelassen. Dennoch gibt es zur fokalen Tonusmodulation bei muskulärer Hypertonie im Kindes- und Jugendalter keine vergleichbaren Behandlungsalternativen, meinte Dr. Sebastian Schröder, München. Mit einer BTX-Therapie sei früh zu beginnen, um auch die Kinder mit Zerebralparese - soweit möglich - an einer normalen Entwicklung der Motorik teilhaben zu lassen. Typisch für diese Kinder ist das Robben auf dem Boden - wenn altersgleiche Kinder bereits Krabbeln. Sie können nur robben, weil sie die Brustund Bizepsmuskulatur nicht ausreichend entspannen können. Dadurch ist es ihnen unmöglich, sich in einem weiteren Schritt ,zu vertikalisieren“. Erst wenn der Bizeps und eventuell die andere Muskulatur medikamentös entspannt werden, sind sie in der Lage, sich hochzudrücken und in die Aufrechte zu gelangen. Krabbeln und die allmähliche Vertikalisie- rung aber geschehen mit sieben oder acht Monaten bis zu einem Jahr. Eine zulassungsgemäße Anwendung von BTX mit zwei Jahren käme hier deutlich zu spät. Es ist für die weitere motorische Entwicklung des Kindes ausreichend, die Symptomatik, hier die Spastik, zu behandeln, damit es in die nächste Entwicklungsphase hineinkommt.

In einem Video zeigte Schröder wie bei einem fast zweijährigen Kind mit Spastik der Beine mit Spitzfußsymptomatik durch die Injektion von BTX ein „schöner Fersenbodenkontakt hergestellt werden konnte“. Das Kind konnte nach nur einer BTX-Gabe innerhalb von drei Monaten frei laufen.

Reimund Freye

6. Deutscher Botulinumtoxin-Kongress,

4. -6.5.2017, Baden-Baden 